Stendhal et l'aristocratie, actes du colloque organisé par "Stendhal aujourd'hui" (25-26 mars 2011), Université Paris-Sorbonne - Paris IV, études réunies et présentées par $\mathrm{M}$. Arrous

\title{
Annalisa Bottacin
}

\section{OpenEdition}

\section{Journals}

Edizione digitale

URL: https://journals.openedition.org/studifrancesi/21366

DOI: $10.4000 /$ studifrancesi.21366

ISSN: 2421-5856

Editore

Rosenberg \& Sellier

\section{Edizione cartacea}

Data di pubblicazione: 1 décembre 2019

Paginazione: 582-584

ISSN: 0039-2944

\section{Notizia bibliografica digitale}

Annalisa Bottacin, «Stendhal et l'aristocratie, actes du colloque organisé par "Stendhal aujourd'hui" (25-26 mars 2011), Université Paris-Sorbonne - Paris IV, études réunies et présentées par M. Arrous», Studi Francesi [Online], 189 (LXIII | III) | 2019, online dal 01 mars 2020, consultato il 12 novembre 2021 URL: http://journals.openedition.org/studifrancesi/21366 ; DOI: https://doi.org/10.4000/studifrancesi. 21366

Questo documento è stato generato automaticamente il 12 novembre 2021.

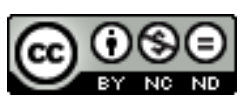

Studi Francesi è distribuita con Licenza Creative Commons Attribuzione - Non commerciale - Non opere derivate 4.0 Internazionale. 


\title{
Stendhal et l'aristocratie, actes du colloque organisé par "Stendhal aujourd'hui" (25-26 mars 2011), Université Paris-Sorbonne - Paris IV, études réunies et présentées par M. Arrous
}

\author{
Annalisa Bottacin
}

\section{NOTIZIA}

Stendhal et l'aristocratie, actes du colloque organisé par "Stendhal aujourd'hui" (25-26 mars 2011), Université Paris-Sorbonne - Paris IV, études réunies et présentées par M. Arrous, Paris, Eurédit, 2018, 317 pp.

1 Nella dotta presentazione, Michel Arrous evidenzia come la tassonomia del ruolo svolto dall'aristocrazia nei romanzi stendhaliani sia oltremodo problematica, in special modo per le differenti fisionomie conferite a tale concetto, come ad esempio allorché definisce la Francia «la nation la moins aristocratique», seppur traspaia sovente a tratti una certa nostalgia per questa specifica classe «quand elle n'est pas étiolée». Il volume si propone di vagliare esaurientemente le reazioni di Stendhal su questo soggetto, esaminando figure e situazioni della sua narrativa. Brahim Karoui in Satire et délégitimation des figures de l'Ancien Régime dans "Le Rouge et le Noir" (pp. 1-24), parte dal presupposto dell'impegno dimostrato da Stendhal nella messa in scena satirica dell'aristocrazia, che per posizione e comportamenti appare una classe destinata a perire, facendo «de son roman une satire en règle des symboles de cette agonie» ed evidenziando il lato menzognero e ironico di una società verso cui Julien Sorel tenta la 
sua scalata, che comunque il suo nobile cuore rifiuta. Claire Deslauriers in Le laconisme aristocratique dans "Lucien Leuwen": discours et attitudes d'une morale naturelle (pp. 25-47), sottolineando la grande fedeltà del romanzo alla Storia, vi legge l'ascesa del giovane Lucien, dagli ideali repubblicani, all'aristocrazia finanziaria degli anni Trenta. L'autrice si sofferma altresì sul ruolo fondamentale giocato dall'uso del corsivo nel romanzo, che sottolinea le diverse funzioni degli aristocratici nel linguaggio e negli atteggiamenti «effaçant par là, le privilège du nom aristocratique, tout en valorisant la noblesse du naturel». Elisabeth Schele in Une relecture de Duclos: Stendhal et la politique aristocratique de la guerre (pp. 49-61) rileva come l'aristocrazia abbia avuto un ruolo di spicco nelle guerre scoppiate all'epoca di Duclos, la cui influenza su Stendhal è stata già ben evidenziata da Jacques Rivain et da Paul Meister, specie nelle pratiche descrittive e nell'aneddoto. Un altro aspetto importante in cui Duclos ha attratto l'attenzione di Stendhal, è aver ben mostrato, attraverso i suoi scritti inerenti alla guerra, la degenerazione del concetto di aristocrazia. Audrey Giboux, De la concurrence des modèles comique et tragique dans "Armance" de Stendhal: la fabrique littéraire d'une exigence de noblesse, de l'héroïsme à la monstruosité (pp. 63-89) scrive di uno Stendhal romanziere realista, come fu definito da Auerbach, depositario di quel «réalisme objectif» ben espresso da George Blin, per la complessità della sua estetica romanzesca, che evidenzia in Armance da un lato i personaggi che incarnano un'aristocrazia dévoilée, secondo le strategie dell'ironia stendhaliana, e dall'altro la rappresentazione di una vera nobiltà di spirito. Armance evidenzia tra l'altro di netto l'ammirazione di Stendhal per il teatro di Molière; in effetti il ritratto degli abitanti del Faubourg Saint-Germain è concepito come una scena teatrale. La dissociazione presente in Armance, tra un'aristocrazia cadente presentata in modo satirico e la nobiltà degli eroi segnati dalla tragedia, è ben rilevata in tutto il romanzo. Françoise Guinoseau in La politique dans "Armance" et "Lucien Leuwen": le règne des salons et des fripons (pp. 153-170) analizza il ruolo svolto dal denaro nell'evoluzione sociale descritta nei romanzi di Stendhal: Mérimée, in una lettera all'amico del maggio 1832, definiva «mauvaise» la società parigina che, pur nelle variazioni sociopolitiche, rimarrà costantemente legata al valore del capitale. Su Armance verte anche lo studio di Sascha Luethy, Le salon aristocratique dans "Armance": un théâtre oculaire (pp. 171-181) che evidenzia l'importanza del sottotitolo, Quelques scènes d'un salon de Paris, un microcosmo che viene a costituire il décor principale del récit, scelto dall'autore per il suo primo romanzo. È ancora Armance l'oggetto di riflessione di Méké Méité, Figure(s) de l'aristocrat(i)e dans "Armance ou Quelques scènes d'un salon de Paris en 1827" (pp. 247-260) in cui il critico si sofferma sull'assiologia dei vari personaggi per meglio situare l'infelice condizione esistenziale del protagonista, Octave de Malivert che oscilla tra due tendenze: «assurer les devoirs liés à son sang et vivre dans son temps». In Les aristocrates à la lanterne? L'aristocratie chez Stendhal et Balzac (pp. 91-105) Max Andréoli costruisce il suo discorso seguendo una linea di diramazione e di convergenza che si fissa in primis su istanze platoniche e sul loro svolgimento nel corso dei tempi, fino all'epoca dei due grandi romanzieri, con particolare riguardo alle tesi di Sant-Simon. Nella sua accurata indagine, Andréoli segnala, con varie esemplificazioni riferite alla loro produzione letteraria, le dissonanze sul concetto di aristocrazia nei due autori. Stendhal la classifica assai raramente; al contrario gli scritti di Balzac abbondano di definizioni, in cui ben si distinguono le varie forme di "aristocratie". Uno Stendhal «aristocrate jacobin» e un Balzac «aristocrate réaliste», pur avendo vari punti di convergenza sulla constatazione della caduta delle élites, si oppongono radicalmente sulle loro «conceptions de l'aristocratie». Anche Brigitte Méra in Aristocratie et jeux de 
Bourse chez Stendhal et Balzac (pp.107-119), prendendo spunto da un'asserzione di Théophile Gautier, tratta dall'Histoire de l'art dramatique en France depuis vingt-cinq ans (1858), in cui viene evidenziato come «les échéances, les coups de Bourse et les grosses sommes ont des moyens d'intérêts tout modernes», analizza finemente come i due scrittori abbiano esaminato il fenomeno dei comportamenti dell'aristocrazia «de l'argent» verso la Borsa che, nel corso dell'Ottocento, con l'affermarsi dell'industrializzazione, si impose quale centro di incontro della domanda e dell'offerta di capitali. In Des aristocraties espagnoles (pp.121-130) Andrée Mansau menziona un incontro di Stendhal a Le Havre, riportato nei Mémoires d'un Touriste, con due giovani spagnole delle quali evidenzia «toute la pétulence [...] toute la coquetterie apparente de la race ibère», da cui scaturisce un paragone con le donne italiane. Sottolinea l'autrice che Stendhal nelle sue novelle trarrà sovente spunto dai costumi della nobiltà della corte napoletana dei Borboni, come «la tragédie des jolies amoureuses dévotes» in Suora Scolastica. In La belle Mme d'Hocquincourt (pp. 131-143), Suzel Esquier disquisisce su un personaggio femminile di Lucien Leuwen, che pur non avendo il ruolo di Mme de Chasteller, è comunque da considerarsi una delle figure di spicco di quella società aristocratica di Nancy che Stendhal dipinge come «une parodie de la cour». Nata in una storica nobiltà, che rinvia a Saint-Simon, dall'educazione altamente aristocratica, Mme d'Hocquincourt si dimostra nei confronti di Lucien molto abile nell'arte del piacere, tanto da suggerire all'autrice per lei il termine di «sprezzatura», usato da Baldassar Castiglione e dal Manzoni, quale atteggiamento ostentatamente disinvolto, da parte di chi si sente molto sicuro dei propri mezzi. Maria Zani in "La Chartreuse de Parme", roman de l'aristocratie (pp. 143-148) vede Stendhal come un aristocratico - giudizio su cui si potrebbe a lungo discutere - il che a suo dire è confermato in tutta la sua opera, in particolar modo nella Chartreuse. Ciò è dovuto al milieu e ai personaggi, a una particolare élite che rappresenterebbe i veri valori romantici, allontanando così atteggiamenti calcolatori ed egoistici in una ricerca di attitudini umanitaristiche e trasparenti. María Vicenta Hernandez Álvarez in L'aristocratie d'une dame courtoise: "La Belle Dame sans merci" et "Le Rouge et le Noir" (pp. 183-209) esordendo con una sottile analisi di De l'amour e soffermandosi sui retaggi che vi si ritrovano tratti dall'Ars amandi di Ovidio e dalla poetica d'André le Chapelain e dei troubadours nei riguardi della dama aristocratica, sottolinea la fondamentale importanza di questo studio sulla fisiologia dell'amore nella creazione dei personaggi femminili, come ad esempio Mme de Rênal che «suit le paradigme de la description de la dame courtoise». Pierrette Pavet in La conversation et la parole aristocratique dans le roman stendhalien (pp. 211-221) evidenzia il grande piacere nutrito da Stendhal per la conversazione, eredità di una tradizione aristocratica d'Ancien Régime, mal imitata e in grande decadenza nei salons della Restaurazione. Dopo una serie di interessanti esemplificazioni, tratte da varie opere, Pavet sintetizza, con una precisa allusione all'epoca di Mme du Deffand, che «le roman stendhalien réinvente cette parole noble en marge de la caste aristocratique». L'interesse di Nicolas Perot si sposta su un amico di Stendhal, Prosper Mérimée e in particolar modo sulla sua celebre novella Carmen ("Carmen" chez la comtesse: l'expérience de la vulgarité, pp. 223-238). La figura di Carmen incarna il fascino erotico esercitato dalla cultura spagnola sugli artisti di espressione francese in epoca romantica, mito brillante e trasgressivo; Perot sottolinea nella stesura di quest'opera l'importanza dell'amicizia tra Mérimée e la madre dell'imperatrice Eugénie de Montijo, Manuela contessa di Teba. Viene infatti riportata la nota lettera, che appare in tutte le edizioni di Carmen, in cui lo scrittore, presentando alla contessa la novella, evidenzia il ruolo 
fondamentale da lei svolto, in quanto si tratta di un soggetto da lei stessa suggerito, nella creazione di questa héroïne bohémienne, dai bassi istinti e dalle provocazioni più ardite. Léda D'Aléo in Quelques sources possibiles pour les personnages de "La Chartreuse de Parme": généalogies et histoires (pp. 239-246) mette in risalto quanto la lettura di Stendhal di opere dei memorialisti, come ad esempio il cardinal de Retz, abbiano ispirato alcuni passaggi dei Mémoires d'un Touriste e, allo stesso modo, un'altra source della Chartreuse de Parme potrebbe essere quella della famiglia napoletana dei Sanseverino, cosicché si giungerebbe anche a sostenere che «le chemin de la Chartreuse passait bien par Naples». In occasione dell'incoronazione di Charles X, avvenuta a Reims nel giugno del 1825, fu commissionata a Rossini una cantata scenica che doveva costituire il culmine dei festeggiamenti dell'importante evento. Con un'idea quantomeno geniale, il librettista Luigi Balocchi propose a Rossini un libretto, che verte su una forzata sosta a Plombières di un gruppo di nobili invitati che, impediti a essere presenti alla cerimonia, improvvisano così una celebrazione nell'albergo, prima di rientrare a Parigi per le festività susseguenti all'incoronazione. Francis Claudon in La Sainte-Alliance de l'Opéra: "Il viaggio a Reims" vs "Le Rouge et le Noir" (pp. 261-272), trae spunto per il suo raffinato intervento dalla cantata rossiniana, evidenziando possibili rapporti con la noblesse del Rouge. Nel bell'articolo conclusivo, il nome di Michel Crouzet è chiaro invito a una profonda meditazione sulla figura del protagonista del Rouge (La formation aristocratique de Julien Sorel, pp. 273-314). L'eminente critico analizza le fasi dell'educazione aristocratica di Julien, dagli inizi a Verrières fino ai salotti parigini dove scopre quanto l'aristocrazia non si faccia scrupoli e quanto poca importanza abbiano il merito e la volontà. «Héros en progrès», Julien riceve all'hôtel de la Mole, «cœur de l'aristostocratie stendhalienne», lezioni di déniaisement, che contribuiscono a estraniarlo dal suo stato, non socialmente ma moralmente. Egli riceve una tale trasformazione che potrebbe stupire da parte di un allievo di Rousseau e Bonaparte. Ma i giochi di Stendhal sono sottilmente raffinati e intimamente profondi... 